\title{
The Role of Invasive Monitoring in Traumatic Brain Injury
}

\author{
Raphael Arellano Carandang ${ }^{1}$
}

Published online: 11 July 2015

(C) Springer International Publishing AG 2015

\begin{abstract}
Severe traumatic brain injury is a complex disease that involves physical injury and distortion of tissues and cell membranes, gross hemodynamic changes including loss of autoregulation, cerebral edema and tissue shifts, changes in pressure and perfusion and multiple secondary cellular processes including electrolyte fluxes, inflammatory mediator release, neurotransmitter mediated excitotoxicity, apoptosis, mitochondrial dysfunction, and alterations in cellular metabolism. The optimal treatment of these patients who have severe neurological dysfunction or require sedation that compromises neurological functional assessment by physical examination requires the monitoring and management of multiple aspects of brain physiology including pressure, perfusion, oxygenation, cellular metabolism, and electrical activity. Invasive monitoring techniques, while still in various stages of development, can and will provide real-time trackable data that informs the management of these patients as well as contributes to our understanding of the pathophysiological processes that contribute to it.
\end{abstract}

Keywords Traumatic brain injury · Invasive multimodal monitoring $\cdot$ Intracranial pressure $\cdot$ Cerebral perfusion pressure $\cdot$ Cerebral blood flow $\cdot$ Oxygenation $\cdot$ Pressure reactivity index $\cdot$ Microdialysis $\cdot$ Near infrared spectroscopy . Laser Doppler flowmetry · Transcranial Doppler ultrasound . $\mathrm{PbtO} 2$

This article is part of the Topical Collection on Blunt Head Trauma

Raphael Arellano Carandang

raphael.carandang@umassmemorial.org

1 Departments of Neurology, Anesthesiology and Surgery, University of Massachusetts Medical School, 55 Lake Avenue North S4-408, Worcester, MA 01655, USA

\section{Introduction}

Traumatic brain injury is all too common and can have devastating consequences and result in severe disability and death. Much research has gone into understanding the numerous gross hemodynamic and physical, biochemical, and cellular processes that occur as the trauma occurs and the consequences thereof that follow [1]. Secondary injury follows cellular membrane damage, resulting in electrolyte fluxes, neurotransmitter release, mitochondrial dysfunction, cytokine and inflammatory mediator release, and apoptotic mechanisms that all contribute to further injury from edema, necrosis, ischemia, metabolic crises, and cell death [2]. Invasive multimodal neurological monitoring is the use of devices to measure, track, and detect pathophysiological changes in the brain after a severe traumatic brain injury with the goal of guiding medical management and therapeutics to help obtain the best possible functional neurological outcome [3].

Many of the principles of management such as the MonroKellie doctrine have been around since the 1800s and from the time of Harvey Cushing, but much of the data following since, until recently, has been slow to accumulate and comes mostly from registries and retrospective database research [4-6]. Given the critical nature of the disease, clinical equipoise for much of the management had been non-existent and guidelines are driven by mostly class II and III level evidence [7, $8 \cdot]$.

Invasive neurological monitoring itself has been on a shifting pendulum in recent years. From measurement of intracranial pressure (ICP) being considered part of the gold standard management and a requirement in the management of patients with severe TBI to being nearly abandoned altogether in some centers because of logistics, cost, perceived risk and lack of evidence. It continues to be surrounded by controversy particularly in light of a recent randomized clinical trial suggesting that it has 
limited impact above and beyond good conventional neurocritical care [9॰]. As with many costly technologies that are in various stages of development, the evidence for its utility continues to grow as does the scrutiny surrounding it. At the current time, it remains largely underutilized [10].

Guidelines $[7,8 \bullet]$ exist, but the impetus for invasive neurological monitoring stems from the physiology of the brain, its sensitivity, and vulnerability to ischemic insults and metabolic crises as well as the need for critical and time-dependent pre-emptive interventions to prevent cell death in the setting of significant brain injury [11]. From a pragmatic standpoint, it is also a necessity given that these patients are often unresponsive, comatose, or require effective sedation to manage their brain injury and whatever accompanying significant polytrauma they suffered in the accident [12]. These patients nearly always require mechanical ventilation and hemodynamic management given their poor airway protection and other organ injuries.

Lastly, invasive monitors provide real-time physiological data and help us further understand the various complex pathophysiological processes that occur in brain injury that in turn inform basic, translational, and clinical research and contribute to the development of treatment protocols as well as therapies.

It is worth emphasizing that all invasive modalities used in monitoring should always be interpreted in light of a comprehensive synthesis of data available in the context of individualized patient care.

\section{ICP Monitoring}

ICP monitoring makes sense from a pathophysiological standpoint as it affects cerebral perfusion pressures and can precipitate or worsen cerebral hypoperfusion and ischemic injury. Clinical studies have not supported this in terms of benefits for outcome. Data from larger observational studies have suggested a threshold of ICP $>20$ for harm, and a meta-analysis of studies has shown this to be associated with worse outcome [13•]. Many guidelines have adopted this as class II evidence for ICP monitoring, but recent studies including a re-analysis of the NTDB data from 1994 to 2001 suggested that ICP monitoring was associated with $45 \%$ lower rate of survival and worse functional outcome with ICP monitoring after correcting for other clinical severity measurements $[14,15]$. The only randomized clinical trial to study an ICP-based treatment protocol with a treatment threshold of 20 versus a conventional one without ICP monitoring for TBI was conducted in South America because of the lack of clinical equipoise in the USA and showed no difference in functional outcome between both groups [9•]. There were some differences in the intensity of the treatment with the non-ICP treatment group getting more hypertonic saline, mannitol, and hyperventilation and more patients in the ICP treatment group getting pentobarbital. The study was a comparison of two protocols and not ICP values per se. If one wanted to truly test the significance of the ICP threshold of 20, they should have inserted ICP monitors in both groups and blinded one group, and upon completion of the trial, un-blinded the nonICP driven protocol group and seen what the ICP values were for that group. What if the ICP numbers were no different in both groups? It does however argue that clinical examination and imaging-driven protocols are just as good as ICP driven ones [12]. More importantly, it is oversimplistic to think that ICP of 20 is a critical threshold that could impact outcome without taking into account multiple other factors such as CPP or autoregulation. This trial has emphasized that the conventional threshold of 20 is not a definitive one and driven more research into continuous ICP measurements, models measuring cerebral autoregulation, revisiting of CPP driven protocols, individualized or patient-specific ICP thresholds, and other multimodal protocols to account for factors other than ICP alone.

\section{CPP-Driven Protocols}

The clinician at the bedside needs to decide between ICP- and CPP-oriented therapy as these often call for different management strategies. CPP-driven protocols require assessments of cerebral autoregulation and monitoring of the ICP response to MAP augmentation. Rosner and colleagues [16] reported favorable outcomes in $35 \%$ of patients with GCS 3 and $75 \%$ of patients with a GCS of 7 in a 158 patient TBI cohort treated using volume expansion, ventriculostomy drainage, mannitol, and pressors using CPP goal $>70$ in 1995. A RCT was conducted by Robertson et al. on 189 patients comparing ICPversus CPP-driven treatment protocols and found that the group with CPP $>70$ had less ischemia when measured by jugular venous desaturations, but outcome results were thought to be diluted by systemic complications of ARDS [17].

\section{Pressure Reactivity Index}

Multiple models have been investigated to access cerebral autoregulation including ones that used arterial-arteriolar cerebrovascular beds, CSF production and resorption, and a resistor mechanism for cerebral veins, another using arterial and venous compartments and CSF and another that uses $\mathrm{ABP}$, ICP, and MCA flow velocities [18-20]. Of these models, the pressure reactivity index (PRx) is the most studied and uses the naturally occurring slow oscillations of $\mathrm{ABF}$ to estimate cerebrovascular reactivity. This is a moving correlation coefficient between 40 consecutive values of ABP and ICP 
averaged over $5 \mathrm{~s}$. Studies [21, 22] have established a correlation between PRx values of $>0.2$ for more than $6 \mathrm{~h}$ and a fatal outcome. Although it has its limitations, it has been found to be a reliable index of cerebral autoregulation validated by TCD and PET and can be used to guide therapy with the calculation of an optimal CPP or an individualized or patient-specific ICP. A single-center study compared conventional ICP thresholds of $20-25$ to ICP thresholds derived from PRx graphs to define ICP doses and found a better correlation of prediction of death than the conventional thresholds in a cohort of 327 patients [23•]. Other models include the pressure-time index which looked at duration and amplitude of the insult and used that to determine critical thresholds of ICP and CPP in children and found to correlate with outcome [24].

Many of these models are using continuous data collected and time and pressure relationship coefficients to improve the sensitivity of predictive thresholds in order to improve the clinical utility of the indices instead of relying on single measurement thresholds.

\section{PbtO2}

Evidence from microdialysis studies as well as preliminary studies using regional oxygen monitoring have found that significant regional hypoxemia and ischemia can occur in the setting of normal ICP and CPP [25]. Studies [26, 27] have since confirmed that low $\mathrm{PbtO} 2$ of less than 10 for $15 \mathrm{~min}$ are associated with worse clinical outcomes and some prospective studies as well as multiple non-randomized studies comparing $\mathrm{ICP} / \mathrm{CPP}$-driven protocols versus ICP/CPP and $\mathrm{PbtO} 2$-driven protocols, and a pooled analysis of these that included 491 patients suggests that there is a twofold likelihood of better outcomes with the $\mathrm{PbtO} 2$ and ICP/CPP-driven protocols [28]. There is still no class 1 evidence, but an ongoing randomized clinical trial in TBI patients will hopefully shed some light on this. The BOOST- 2 phase 2 randomized trial for safety and efficacy found that using a $\mathrm{PbtO} 2$ and ICP/CPP-driven protocol reduced the amount of time that regional ischemia occurred, and there was no significant difference in adverse events [29]. There was lower overall mortality and poor outcome in the $\mathrm{PbtO} 2$ and ICP/CPP-driven group which has led to phase 3 which is currently recruiting patients. There are still multiple unanswered questions and including when to treat, how best to treat, and the significance of self-limited episodes of low regional oxygenation that correct without intervention.

\section{SjVO2}

$\mathrm{SjvO} 2$ measures global hypoxemia when inserted into the dominant jugular vein but more specifically can be used as a measure of oxygen delivery and consumption. $\mathrm{CMRO} 2=\mathrm{CBF}$ $(\mathrm{CaO} 2-\mathrm{CvO} 2)$. This is an index of oxygen utilization and consumption and thresholds of $<50 \%$ lasting for $15 \mathrm{~min}$ or more reflect poor oxygen delivery and ischemia and have been found to be associated with poor neurological outcome [30]. Conversely, values of $>80 \%$ may reflect poor oxygen utilization, decreased metabolic demand, or hyperemia [31]. $\mathrm{SjvO} 2$ are not often utilized given multiple concerns of poor sensitivity (requiring significant ischemia before detection), technical issues with maintaining proper positioning, calibration and questions of accuracy, the invasive nature of the risks of complications including carotid puncture, pneumothorax, bleeding, and infection but especially jugular venous thrombosis while uncommon may have serious consequences in a patient with already significant cerebral edema. The author and some other centers utilize the central venous catheter as venous saturation values have been found to be comparable although not exactly numerically equivalent, and many severe TBI patients will need central lines for various reasons [32]. The major limitation, however, is the fact that saturations can vary minute to minute, and blood sampling only affords the clinician a single snapshot making repeated sampling necessary or continuous monitoring with oximetry preferred [33]. Although the utility of central venous oxygen saturation monitoring has been called into question by the ProCESS trial for sepsis [34], and the utility of central venous $\mathrm{O}_{2}$ saturations is controversial, many intensivists including this author will utilize it in the context of synthesis of multiple data points in the individualized patient.

\section{Microdialysis}

Cerebral microdialysis is unique among all other multimodal monitoring devices in that it allows assessment of neurochemical processes that occur in traumatic brain injury and other brain injury disease states. A microdialysis catheter is inserted into the brain tissue, and diffusion of molecules across a semipermeable dialysis membrane allows the collection of various substances from the extracellular fluid and subsequent analyses [35]. Much of the analyses currently being studied involves biomarkers of bioenergetics, excitotoxicity, and cell degeneration and provides insight into cell metabolism in the setting of ischemia and hypoglycemia providing further information into the cellular crisis that occurs in secondary injury from traumatic brain injury [36]. Clinical correlative data exists for lactate: pyruvate ratios, glucose, glycerol, and glutamate levels that have been found to be predictive of intracranial hypertension or relative ischemia or infarction in the setting of vasospasm as well as with outcome. Lactate: pyruvate ratios reflect anaerobic glycolysis secondary to ischemia and mitochondrial failure with ratios $>20-25$ being associated with poor outcome in TBI [36-38]. Glycerol levels of 
$>100 \mu \mathrm{mol} / \mathrm{L}$, glucose levels $<1.5-2 \mathrm{mmol} / \mathrm{L}$, and glutamate levels $>15-20 \mathrm{~mol} / \mathrm{L}$ are also associated with poor outcome and reflect metabolic crisis in TBI [39]. This technique has allowed the exploration of other biomarkers such as neurofilament heavy chain which, in one study, had an OR 7.68 for predicting mortality in TBI which was superior to ICP, GCS, and CPP [40 $]$ and Tau levels which, in another preliminary study, were found to correlate with MRI DTI and may be a possible marker for traumatic axonal injury [41•]. It also allows exploration of the relationships between lactate levels and hypoxemia to further elucidate mechanisms of injury and characterize them, for example, as mitochondrial failure versus ischemia [42] and combined with PET and MR SPECT scan, a better understanding of glucose metabolism following traumatic brain injury [38]. Microdialysis provides continuous, real-time assays of ECF fluid metabolites but needs to be interpreted in light of the complex processes that affect concentration changes, including the effect of the injury, type, location and severity, and drugs such as sedation and anesthesia that may affect brain metabolism and influence these levels [43]. Given the complexity of this data, the logistical requirements, and invasive nature of this method, clinical use is currently still limited to academic research centers that have the funding and resources to utilize it.

\section{Cerebral Blood Flow}

Other than imaging techniques such as Xenon CT, CTA and perfusion, and MR perfusion, all of which do not provide continuous bedside monitoring, there are limited devices to monitor cerebral blood flow. Transcranial Doppler ultrasound is a non-invasive bedside tool to measure blood flow indirectly by velocities and can be accurate in measuring cerebral perfusion as well as cerebral autoregulation in TBI patients with good correlation with PET studies [44], but has limited resolution, only visualizing large vessels, is highly operator dependent, and suffers from logistical issues such as consistency of probe positioning variable bone windows and computer interfacing [45]. Laser Doppler flowmetry uses the same principle as TCD but has the probe placed intracranially through a burr hole and measures the concentration of red blood cells and their velocity generating a flow signal to provide a qualitative estimate of regional cerebral blood flow but has a limited sample volume [46]. The thermal diffusion method utilizes an intraparenchymal probe with a thermistor and a temperature sensor and measures the thermal gradient between the distal thermistor that his heated by $2{ }^{\circ} \mathrm{C}$ and the proximal temperature sensor and provides a quantified regional cerebral blood flow measurement in $\mathrm{ml} / 100 \mathrm{~g} / \mathrm{min}$ [47]. CBF monitoring has been studies in traumatic brain injury, and thresholds of injury and ischemia have been found to correlate with regional brain tissue oxygenation. Certain thresholds are thought to correlate with ischemia as well as vasospasm, but this technology is not in widespread use clinically. Preliminary small studies have been done to assess its usefulness and reliability in measuring autoregulation combined with ICP, CPP, and PRx and found it to be safe with minimal complications and able to provide measurements of local vascular resistance [48]. Limitations include effects of temperature and hyperthermia, and there is no data correlating with clinical outcomes at this time.

Other non-invasive methods of measuring cerebral blood flow and cerebral autoreactivity include near infrared spectroscopy (NIRS) [49] and direct correlation spectroscopy (DCS) [50] both of which utilize infrared spectrum light to penetrate tissues and the different photic properties of chromophores such as oxy and de-oxyhemoglobin and their temporal intensity fluctuations to measure oxygen saturation and with the use of contrast agents such as indocyanine green or fluorescent sphere tracers, changes in tissue blood flow, and microvascular CBF. These are currently still being studied and validated in other brain injury states such as ischemic stroke, subarachnoid hemorrhage, and perioperatively in cardiac and carotid surgery but have limited clinical utility in traumatic brain injury as of this writing.

\section{Continuous EEG Monitoring and Electrocorticography}

Although surface EEG is considered non-invasive, it qualifies as a physiological bedside monitor of brain function and electrical activity and has a definite role in the monitoring of traumatic brain injury patients who are critically ill. The most common indication for EEG monitoring is neurological fluctuations on examination and to rule out non-convulsive status epilepticus which is a treatable cause of unresponsiveness or impaired consciousness in the traumatic brain injury patient [51]. This can help guide therapy with anticonvulsant medications which can also have multiple and significant adverse effects such as fever, hemodynamic changes such as hypotension, delirium, encephalopathy, and somnolence in patients who have temporal lobe contusions, subdural hematomas, or depressed skull fractures which may have a higher risk of seizures. From a prognostication standpoint, EEG has also been found to correlate with clinical outcomes in traumatic brain injury patients with reduced percent alpha variability (PAV) being a key predictor of poor functional clinical outcome at 30 days and 6 months post-trauma as well as thalamic involvement [52]. The alpha-delta ratio has also been studied in small cohorts and been found to correlate with outcome after rehabilitation [53].

Electrocorticography is a more invasive form of EEG monitoring where recording grids and strips are laid on the cortical surface intraoperatively. This technique affords more sensitive 
Table 1 Multimodal invasive neurological monitoring. Critical thresholds, data collected studies supporting use, and issues

\begin{tabular}{|c|c|c|c|c|}
\hline Mode & Critical Thresholds & Data & Studies & Issues \\
\hline ICP & $>25$ & Pressure & $\begin{array}{l}\text { Marmarou 1991, Cremer } 2005 \\
\text { Shafi 2009, Chestnut } 2012\end{array}$ & RCT negative unclear threshold \\
\hline CPP & $<60$ and $>70$ & Perfusion & Rosner 1995, Robertson 1999 & Increased risk of ARDS \\
\hline PRx & $>0.2$ & Autoregulation & Steiner 2002, Howells 2005 & Large data, calculations and processing \\
\hline $\mathrm{PbtOZ}$ & $<10-25$ & Regional oxygen & $\begin{array}{l}\text { Narotam 2009, Spiotta 2010, } \\
\text { Steifel 2005, }\end{array}$ & $\begin{array}{l}\text { Unclear best location for placement } \\
\text { Requires calibration }\end{array}$ \\
\hline $\mathrm{SjvOZ}$ & $\begin{array}{l}<50 \% \\
>80 \%\end{array}$ & $\begin{array}{l}\text { Global oxygen } \\
\text { Oxygen utilization }\end{array}$ & $\begin{array}{l}\text { Gopinath } 1994 \\
\text { Cruz } 1993\end{array}$ & $\begin{array}{l}\text { Risk of venous thrombosis, durability } \\
\text { Need for recalibration, gaps In data }\end{array}$ \\
\hline \multirow[t]{4}{*}{ MD } & Glucose $<2.0$ & Decreased perfusion & Schulz 2000, Vespa 2003 & $\begin{array}{l}\text { Not widely available, logistical } \\
\text { requirements }\end{array}$ \\
\hline & $1 / \mathrm{P}$ ratio $>20-25$ & Metabolic crisis/ischemia & $\begin{array}{l}\text { Hillered 1996, Robertson 1998, } \\
\text { Valadka 1998 } \\
\text { Zauner 1997, Nordstrom 2003, } \\
\text { Stahl 2001 }\end{array}$ & $\begin{array}{l}\text { Selection of biomarkers, quantification } \\
\text { Computer interfacing, } \\
\text { presentation of data }\end{array}$ \\
\hline & Glut $>15-20$ & Excitotoxicity & Bullock 1995, Hutchinson 2000 & Implantation strategies \\
\hline & Glycerol $>100$ & Cell membrane degradation & $\begin{array}{l}\text { Marklund 1997, Hillered 1998, } \\
\text { Reinstrup } 2000\end{array}$ & Standardization of protocols \\
\hline \multirow[t]{2}{*}{$\mathrm{CBF}$} & $<18 \mathrm{mi} / 100 \mathrm{~g} / \mathrm{min}$ & Ischemia & Thome 2001, Jaeger 2005 & Needs validation, limited by temperature \\
\hline & $<15 \mathrm{ml} / \mathrm{g} / \mathrm{min}$ & Vasospasm & Vajkoczy 2003 & Frequent calibration \\
\hline NIRS & $\mathrm{rSO} 2<55 \%$ & Ischemia & Untenberg 1995 & Spatial resolution, Needs validation \\
\hline \multirow[t]{2}{*}{ EEG } & $\mathrm{PAV}<0.1$ & Electrical activity & Vespa 2002 & Large data, analytic software needed \\
\hline & $\mathrm{ADR}>10 \%$ & Vasospasm/DCI & $\begin{array}{l}\text { Claasen 2004, Finnigan 2007, } \\
\text { Leon-Carrion } 2009\end{array}$ & Scalp fixation, artifacts, cumbersome \\
\hline ECoG & Depolarizations & Cortical spreading depression & Hartings 2009, Hartings 2011 & $\begin{array}{l}\text { Cumbersome, Analytk software, } \\
\text { large data }\end{array}$ \\
\hline
\end{tabular}

$I C P$ intracranial pressure, $C P P$ cerebral perfusion pressure, $\mathrm{PRx}$ pressure reactivity index, $\mathrm{PbtO} 2$ partial pressure of brain tissue oxygenation, $\mathrm{Sj} V \mathrm{O} 2$ jugular venous $\mathrm{O}_{2}$ saturation, $M D$ microdialysis, $C B F$ cerebral blood flow, $N I R S$ near infrared spectroscopy, $E E G$ electroencephalography, $E C o G$ electrocorticography

readings than surface EEG and allows the detection of areas of cortical spreading depression. Spreading depolarizations have been found to correlate with clinical outcome and be predictive of metabolic crisis, increased intracranial pressure and vasospasm, and delayed cerebral ischemia [54]. Prolonged depolarizations with negative direct current shifts were associated with isoelectric or periodic epileptiform discharges, prolonged depression of spontaneous activity and occurrence in temporal clusters, and are thought to reflect poor tissue perfusion and/or neurovascular uncoupling and correlated with a worse prognosis [55]. A simple scoring method provided graded prognosis corresponding to $100 \%$ isoelectric depolarizations, $60 \%$ depolarization with depressed periods, and $23 \%$ no depolarization of poor outcomes.

\section{Conclusion}

There are numerous and rapidly developing technologies for invasive neurological monitoring (see Table 1); some have been available for decades and continue to be considered useful mainstays of management of severe acute traumatic brain injury such as ICP, CPP, BP, oxygenation and $\mathrm{pCO} 2$, and temperature. Others are recently coming into clinical practice with evolving clinical data to support their use such as $\mathrm{PbtO} 2$ and EEG while others remain mostly research tools in academic centers such as electrocorticography, CBF, NIRs, DCS, and microdialysis, but are being vigorously studied and together with neuroimaging studies such as PET, MRSP ECT, MRI DTI, and Xenon CT are contributing significantly to our understanding of the secondary pathophysiological, electrical, neurochemical, and metabolic processes that occur in traumatic brain injury and spurring further basic, translational, and clinical research in the field. Clinical outcome data is also available and forthcoming but not yet definitive for benefit which is why there continues to be controversy in utilization of these technologies.

When the BEST-Trip trial results [9॰] were reported, many were not surprised that there was no difference in outcomes for ICP-driven protocols because traumatic brain injury is a complex and heterogeneous disorder and our patients are complicated with a myriad of things that factor into their 


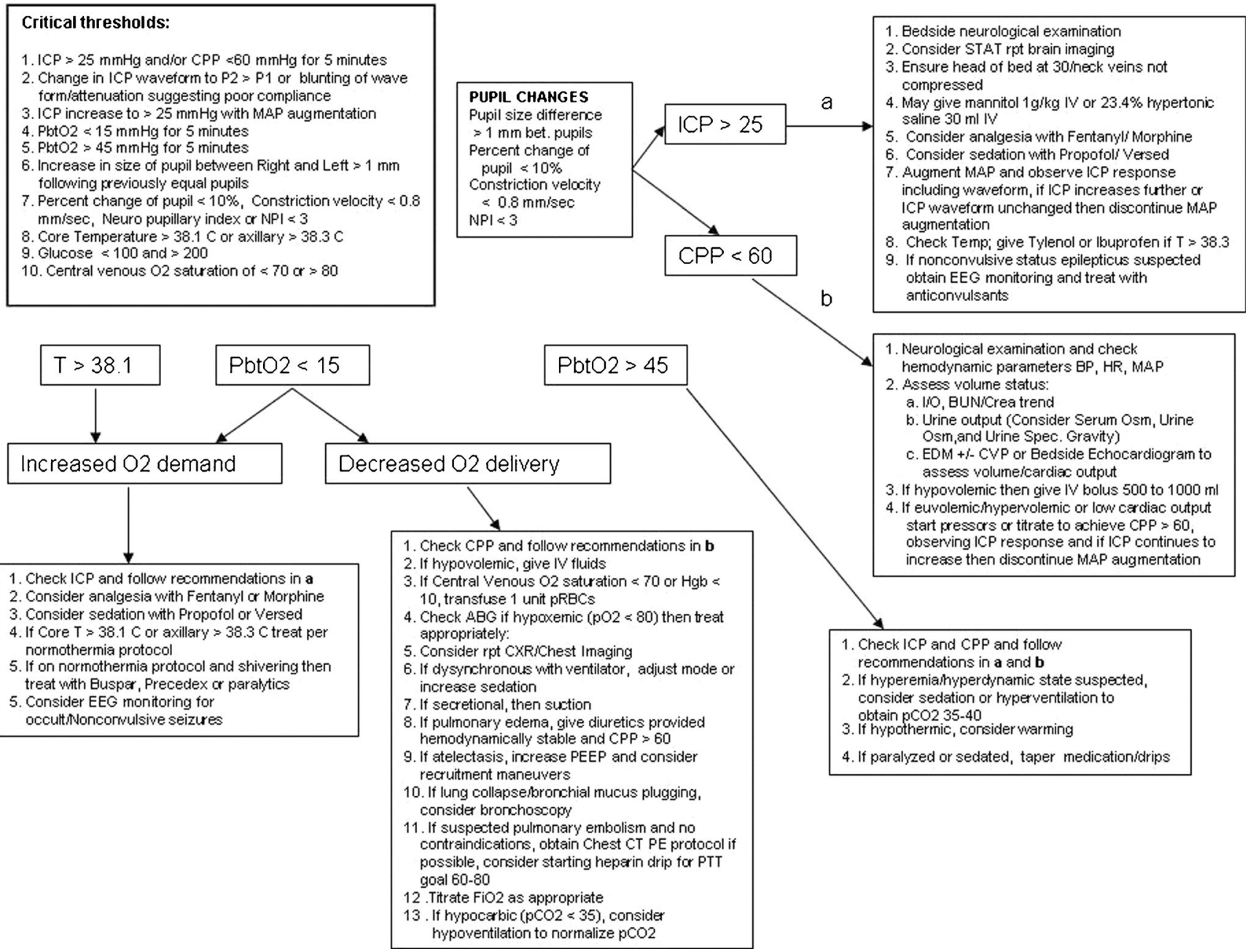

Fig. 1 Example of a multimodal monitoring protocol in current use in a level 1 academic trauma center

outcome so that it would be quite naïve to think that a single pressure value would make the difference in outcome. That's why much of the newer literature calls for combining multiple modes of monitoring into a more comprehensive monitoring schema and many centers have formulated multimodal monitoring protocols that attempt to address and optimize the multiple physiological parameters that affect brain function (Fig. 1).

It helps to remember that critical care and especially neurocritical care are evolving fields of practice that deal with complicated disorders of the most complex and still not a wellunderstood system of the body in the brain and nervous system. Many view the development of these technologies and multimodal monitoring systems as our acknowledgement of the complexities of the brain and its response to injury and our attempt to grapple with the numerous and multifaceted aspects and physiological parameters that go into the care of these patients in order to better understand them and improve our therapeutics and prognostication with the ultimate goal of improving outcomes.

\section{Compliance with Ethics Guidelines}

Conflict of Interest Dr. Carandang declares no conflict of interest.

Human and Animal Rights and Informed Consent This article does not contain any studies with human or animal subjects performed by any of the authors.

\section{References}

Papers of particular interest, published recently, have been highlighted as:

- Of importance

1. Maas AIR, Stocchetti N, Bullock R. Moderate and severe traumatic brain injury in adults. Lancet Neurol. 2008;7:728-41.

2. Werner C, Engelhard K. Pathophysiology of traumatic brain injury. Br J Anaesth. 2007;88:4-9. 
3. Stocchetti N, Le Roux P, Vespa P, Oddo M, Citerio G, Andrews PK, et al. Clinical review: neuromonitoring an update. Crit Care. 2013;17:201.

4. Mokri B. The Monro-Kellie hypothesis: applications in CSF volume depletion. Neurology. 2001;56:1746-8.

5. Cushing $\mathrm{H}$. The blood pressure reaction of acute cerebral compression, illustrated by cases of intracranial hemorrhage. Am J Sci. 1903;125:1017-44.

6. Marshall LF, Toole BM, Bowers SA. The National Traumatic Coma Data Bank. J Neurosurg. 1983;59:285-88.

7. Bullock R et al. Guidelines for the management of severe traumatic brain injury. J Neurotrauma. 2007;24:S1-106.

8. Le Roux P et al. Consensus Summary Statement of the International Multidisciplinary Consensus Conference on Multimodality Monitoring in Neurocritical Care. A statement for health care professionals from the Neurocritical Care Society and the European Society of Intensive Care Medicine. Neurocrit Care. 2014;21(Supplement 2):S1-S297. A Comprehensive compendium of review articles including evidentiary tables on all modes of monitoring from multidisciplinary and multinational specialists and authorities on the topics.

9. Chesnut RM, Temkin N, Carney N, Dikmen S, Rondina C, Videtta $\mathrm{W}$, et al. A trial of intracranial-pressure monitoring in traumatic brain injury. N Engl J Med. 2012;367:2471-81. Recent Level I evidence for ICP monitoring protocol versus Clinical-Imaging protocol which served as the impetus for re-thinking multimodal monitoring and addressing its complexity.

10. Hesdorffer D, Ghajar J, Iacono L. Predictors of compliance with evidence based guidelines for traumatic brain injury care: a survey of United States Trauma Centers. J Trauma. 2002;52:1202-9.

11. Carandang R, Hall WR, Prough DS. Neurologic multimodal monitoring. In: Irwin RS, Rippe JM, editors. Irwin and Rippe's intensive care medicine, 7th ed. Philadelphia: Lipincott, Williams and Wilkins; 2012. Chapter 28, p. 258-271.

12. Ropper AH. Brain in a box. Editorial. N Engl J Med. 2012;367: 2259-541.

13. Marmarou A, Anderson RL, Ward JD, Choi SC, Young HF, Eisenberg HM, et al. Impact of ICP instability and hypotension on outcome in patients with severe head trauma. J Neurosurg. 1991;75 Suppl 1:S59-66. Landmark study from National Traumatic Coma Data Bank which made ICP monitoring an important standard of care. A good illustration of analysis of retrospective data base/registries.

14. Shafi S, Diaz-Arrastia R, Madden C, Gentilello L. Intracranial pressure monitoring in brain-injured patients is associated with worsening of survival. J Trauma. 2008;64:335-40.

15. Cremer OL et al. Effect of intracranial pressure monitoring and targeted intensive care on functional outcome after severe head injury. Crit Care Med. 2005;33:2207-13.

16. Rosner MJ, Rosner SD, Johnson AH. Cerebral perfusion pressure: management protocol and clinical results. J Neurosurg. 1995;83: 949-62.

17. Robertson CS, Valadka AB, Hannay JH, et al. Prevention of secondary ischemic insults after severe head injury. Crit Care Med. 1999;27:2086-95.

18. Kim DJ, Czosnyka M, Keong N, et al. Index of cerebrospinal compensatory reserve in hydrocephalus. Neurosurgery. 2009;64:494-502.

19. Aries MJ, Czosynka M, Budohoski KP, et al. Continuous determination of optimal cerebral perfusion pressure in traumatic brain injury. Crit Care Med. 2012;40:2456-63.

20. Vik A, Nag T, Fredriksli OA, Skandsen T, Moen KG, SchirmerMikalsen K, et al. Relationship of "dose" of intracranial hypertension to outcome in severe traumatic brain injury. Clinical Article. J Neurosurg. 2008; 109:678-84.

21. Howells T, Elf K, Jones PA, Ronne-Engström E, Piper I, Nilsson P, et al. Pressure reactivity as a guide in the treatment of cerebral perfusion pressure in patients with brain trauma. J Neurosurg. 2005;102:311-7.

22. Steiner LA, Czosnyka M, Piechnik SK, et al. Continuous monitoring of cerebrovascular pressure reactivity allows determination of optimal cerebral perfusion pressure in patients with traumatic brain injury. Crit Care Med. 2002;30:733-8.

23. Lazardis C, DeSantis SM, Smielewski P, Menon DK, Hutchinson P, Pickard JD, et al. Patient specific thresholds of intracranial pressure in severe traumatic brain injury. J Neurosurg. 2014;120(4):893900. Important paper that emphasizes individualized therapy using Pressure reactivity index that found good correlation with outcome.

24. Chambers IR, Jones PA, Lo TYM, et al. Critical thresholds for ICP and CPP in pediatric head injury. JNNP. 2006;77:234-40.

25. Meixensberger J, Jaeger N, Vath A, et al. Brain tissue oxygen guided treatment supplementing ICP/CPP therapy after traumatic brain injury. JNPP. 2003;74:760-4.

26. Narotam PK, Morrison JF, Nathoo N. Brain tissue oxygen monitoring in traumatic brain injury, major trauma outcome analysis of brain tissue oxygen-directed therapy. J Neurosurg. 2009;111:672-82.

27. Spiotta AM, Stiefel MF, Gracias VH, et al. Brain tissue oxygen directed management, outcome in patients with severe traumatic brain injury. J Neurosurg. 2010;113:571-80.

28. Nangunoori R, Maloney-Wilensky E, Stiefel M, et al. Brain tissue oxygen based therapy and outcome after severe traumatic brain injury: a systematic literature review. Neurocrit Care. 2012;17:131-8.

29. Shutter L for BOOST-2 Investigators. Preliminary results of Brain Tissue Oxygen Monitoring in Traumatic Brain Injury (BOOST-2) Trial. Oral presentation of Breaking Clinical Trial abstracts presented at Annual Meeting of Neurocritical Care Society, 2014, October 17th, Seattle, WA, ClinicalTrials.gov NCT00974259.

30. Gopinath SP, Robertson CS, Contant CF, et al. Jugular venous desaturations and outcome in traumatic brain injury. JNNP. 1994;57:717-23.

31. Cruz J, Jaggi JL, Hoffstad OJ. Cerebral blood flow and oxygen consumption in acute brain injury with anemia: an alternative for the cerebral metabolic rate of oxygen consumption? Crit Care Med. 1993;21:1218-24

32. Walley KR. Use of central venous oxygen saturation to guide therapy. Am J Respir Crit Care Med. 2011;184(5):514-20.

33. Cruz J. The first decade of continuous monitoring of jugular bulb oxyhemoglobin saturation: management strategies and clinical outcome. Crit Care Med. 1998;26:344-51.

34. The ProCESS investigators. A randomized trial of protocol-based care for early septic shock. N Engl J Med. 2014;370:1683-93.

35. Johnston AJ, Gupta AK. Advanced monitoring in the neurology intensive care unit: microdialysis. Curr Opin Crit Care. 2002;30: 1062-70.

36. Hillered L, Vespa PM, Hovda DA. Translational neurochemical research in acute human brain injury: the current status and potential future for cerebral microdialysis. J Neurotrauma. 2005;22:3-41.

37. Andrews OJ, Citerio G, Longhi L. NICEM consensus on neurological monitoring in acute neurological disease. Intensive Care Med. 2008;34:1362-70.

38. Bellander BM, Cantais E, Enblad P, et al. Consensus meeting on microdialysis in neurointensive care. Intensive Care Med. 2004;30: 2166-9.

39. Petzold A, Tisdall MM, Girbes AR, Martinian L, Thom M, Kitchen $\mathrm{N}$, et al. In vivo monitoring of neuronal loss in traumatic brain injury: a microdialysis study. Brain. 2011;143:464-84.

40. Magnoni S, MacDonald CL, Esparza TJ, Conte V, Sorrell J, Macri $\mathrm{M}$, et al. Quantitative assessments of traumatic axonal injury in human brain: concordance of microdialysis and advanced MRI. Brain. 2015. doi:10.1093/brain/awv152. Recent paper illustrating how combining modern imaging studies with microdialysis can lead to biomarkers that further inform our 
understanding of the pathophysiology of traumatic brain injury.

41. Lazaridis C, Andrews C. Brain tissue oxygenation, Lactatepyruvate ratio, and cerebrovascular pressure reactivity monitoring in severe TBI: a systematic review and viewpoint. Neurocrit Care. 2014;21:345-55. Good review of combined modalities and their improved utility in monitoring.

42. Jalloh I, Carpenter KL, Hely A, et al. Glucose metabolism following human TBI methods of assessment and pathophysiological findings. Met Brain Dis. 2015;30:615-32.

43. Steiner LA, Coles JP, Johnston AJ, et al. Assessment of cerebrovascular autoregulation in head injury patients: a validation study. Stroke. 2003;34:2404-9.

44. Czosnyka M, Smielewski P, Piechnik S, et al. Cerebral autoregulation following head injury. J Neurosurg. 2001;95:756-63.

45. Miller C, Armonda R. Monitoring of cerebral blood flow and ischemia in the critically ill. Neurocrit Care. 2014;21:121-8.

46. Lam JMK, Hsiang JNK, Poon WS. Monitoring of autoregulation using laser Doppler flowmetry in patients with head injury. J Neurosurg. 1997;86:438-45.

47. Wajkoczy P, Roth H, Horn P, et al. Continuous monitoring of regional cerebral blood flow: experimental and clinical validation of a novel thermal diffusion microprobe. J Neurosurg. 2000;93:265-74.

48. Rosenthal G, Sanchez-Mejia R, Phan N, Hemphill JC, Martin C, Manley GT. Incorporating a parenchymal thermal diffusion cerebral blood flow probe in bedside assessment of cerebral autoregulation and vasoreactivity in patients with severe traumatic brain injury. $\mathrm{J}$ Neurosurg. 2011;114:62-70.

49. Leal-Noval SR, Cayuela A, Rellano-Orden V, et al. Invasive and noninvasive assessment of cerebral oxygenation in patients with severe traumatic brain injury. Intensive Care Med. 2010;36:1309-17.

50. Kim MN, Durduran T, Frangos S, et al. Noninvasive measurement of cerebral blood flow and blood oxygenation using near-infrared and diffuse correlation spectroscopies in critically brain injured adults. Neurocrit Care. 2010;12:173-80.

51. Claassen J, Mayer SA, Kowakski RG, et al. Detection of electrographic seizures with continuous EEG monitoring in the critically ill patients. Neurology. 2004;62:1743-8.

52. Vespa PM, Boscardin WJ, McArthur DL, et al. Early and persistent impaired percent alpha variability on continuous electroencephalography monitoring as predictive of poor outcome after traumatic brain injury. J Neurosurg. 2002;97:84-92.

53. Hebb MO, McArthur DL, Alger J, et al. Impaired percent alpha variability on continuous electroencephalography is associated with thalamic injury and predicts poor long-term outcome after human traumatic brain injury. J Neurotrauma. 2007;24:579-90

54. Dreier JP. The role of spreading depression, spreading depolarization and spreading ischemia in neurological disease. Nat Med. 2011;17:439-47.

55. Hartings JA, Watanabe T, Bullock MR, et al. Spreading depolarizations have prolonged direct current shifts and are associated with poor outcome in brain trauma. Brain. 2011;134:1529-40. 